\title{
Crystal growth and optical characterization of terbium and niobium doped BTO single crystals
}

\author{
T. O. dos Santos ${ }^{1 *}$, R. Montenegro ${ }^{1}$, A. W. Oliveira ${ }^{2}$ and J. F. Carvalho ${ }^{1}$ \\ ${ }^{1}$ Instituto de Física, Universidade Federal de Goiás, Av. Esperança, s/nº , Campus Samambaia, 74690-900, \\ Goiânia, GO, Brasil. \\ ${ }^{2}$ Universidade Federal do Tocantins, Av. NS15 ALCNO 14, 77000-000, Palmas, TO, Brasil \\ ${ }^{(*)}$ E-mail: tatiane oliveira@ufg.br
}

Received: 01/12/2016 Accepted: 11/09/2017

DOI: $10.7149 /$ OPA.50.4.49031

\begin{abstract}
:
In this study, single crystals of $\mathrm{Bi}_{12} \mathrm{TiO}_{20}$ doped with niobium and terbium were grown by Top Seeded Solution Growth technique (TSSG). Samples with good optical quality were obtained and were investigated concerning optical absorption, optical activity, electro-optic effect, and photoconductivity. It was verified that doping with terbium and niobium changes the defects structure characteristic of sillenites, affecting in different ways the optical behavior of crystals.
\end{abstract}

Key words: Sillenite Crystals, Photorefractive Materials, BTO, Niobium Doping, Terbium Doping

\section{REFERENCES AND LINKS}

[1] J. Frejlich, Photorefractive Materials: Fundamental Concepts, Holographic Recording and Materials Characterization, John Wiley \& Sons (2007).

[2] D. Bloom, S.W.S. McKeever, "Trapping of Photocarriers in Ga-Doped $\mathrm{Bi}_{12} \mathrm{GeO}_{20}$," J. Appl. Phys. 77, 6511-6520 (1995).

[3] T. O. dos Santos, J.F. Carvalho, A. C. Hernandes, "Synthesis and crystal growth of sillenite phases in the $\mathrm{Bi}_{2} \mathrm{O}_{3}-\mathrm{TiO}_{2}-\mathrm{Nb}_{2} \mathrm{O}_{5}$ system," Cryst. Res. Technol. 39, 10, 868-872 (2004).

[4] J. Frejlich, R. Montenegro, T. O. dos Santos, J.F. Carvalho, "Characterization of photorefractive undoped and doped sillenite crystals using holographic and photoconductivity techniques" J. Opt. A: Pure Appl. Opt. 10 (2008).

[5] J. F. Carvalho, A. C. Hernandes, "Growth and optical characterization of cerium and lead-doped $\mathrm{Bi}_{12} \mathrm{TiO}_{20}$ sillenite single crystals," Cryst. Res. Technol. 40, 9, 847-851 (2005).

[6] M. Bryushinin, V. Kulikov, I. Sokolov, P. Delaye, and G. Pauliat, "Non-steady-state photoelectromotive force and two-wave mixing in photorefractive crystals under frequency modulated illumination". Journal of the Optical Society of America B 31, 723-729 (2014).

[7] V. Marinova, R. C. Liu, S. H. Lin, M-S Chen, Y. H. Lin, K. Y. Hsu, "Near-infrared properties of Rh-doped $\mathrm{Bi}_{12} \mathrm{TiO}_{20}$ crystals for photonic applications". Optics Letters 38, 495-497 (2013).

[8] F. Mersch, K. Buse, W. Sauf, H. Hesse, and E. Krätzig, "Growth and Characterization of Undoped and Doped $\mathrm{Bi}_{12} \mathrm{TiO}_{20}$ Crystals," Phys. Stat. Sol. (a) 140, 273 (1993).

[9] J. F. Carvalho, A. C. Hernandes, "Large $\mathrm{Bi}_{12} \mathrm{TiO}_{20}$ single crystals: a study of intrinsic defects and growth parameters," Journal of Crystal Growth, 205, 185-190 (1999).

[10] R. Oberschmid, "Absorption centers of $\mathrm{Bi}_{12} \mathrm{GeO}_{20}$ and $\mathrm{Bi}_{12} \mathrm{SiO}_{20}$ crystals," Phys. Stat. Sol. (a), 89, 263 (1985).

[11] B.C.Grabmaier and R.Oberschmid, "Properties of pure and doped $\mathrm{Bi}_{12} \mathrm{GeO}_{20}$ and $\mathrm{Bi}_{12} \mathrm{SiO}_{20}$ crystals", Physica Status Solidi (a), 96, 199-210 (1986).

[12] M. Henry, S. Mallick, D. Rouède, "Propagation of light in an optically active electro-optic crystal of $\mathrm{Bi}_{12} \mathrm{SiO}_{20}$ : Measurement of the electro-optic coefficient," J. Appl. Phys, 59, 2650 (1986).

[13] I. de Oliveira, T. O. dos Santos, J. F. Carvalho, J. Frejlich, "Electro-optic coefficient and wavelength dispersion in sillenite crystals," Applied Physics B, 105, 2, 301-304 (2011).

[14] R. Montenegro, N. R. Inocente-Junior, J. Frejlich, "New possibilities for the measurement of wavelength-resolved photoconductivity," Review of Scientific Instruments 77, 043905 (2006). 


\section{Introduction}

Sillenite crystals, having compositions $\mathrm{Bi}_{12} \mathrm{MO}_{20}$, where $\mathrm{M}=\mathrm{Ti}, \mathrm{Si}, \mathrm{Ge}$ and other, exhibit a number of interesting properties such as optical activity, piezoelectricity, photoconductivity, and linear electrooptical effect. The combination of photoconductivity with the linear electro-optical effect results in the socalled photorefractive effect, that consist of a reversible light-induced change in the refractive index of the crystal [1]. The photorefractive effect observed in these materials is determined by the transport dynamics associated with the photogeneration and trapping of electronic charge carriers in localized centers in the crystal bandgap [2]. Being intrinsically related to the localized centers, the performance of photorefractive materials can be altered by doping, and eventually improving it with suitable impurities.

In sillenite family, $\mathrm{Bi}_{12} \mathrm{TiO}_{20}$ (BTO) crystals present some practical advantages for photorefractive applications, such as lower optical activity, higher electro-optic coefficient, higher photoconductivity and increased sensitivity to red light [1], and the influence of impurities on the properties affecting the photorefractive response of BTO has been subject of extensive research [3-7]. However, the search for new compositions resulting in crystals with improved photorefractive response remains as a present challenge.

The present study is concerned to the growth of BTO crystals doped with terbium and niobium, and the investigation of their optical properties by optical absorption, optical activity, electro-optic, and photoconductivity measurements.

\section{Crystal Growth}

The terbium and niobium doped BTO crystals were grown using the Top Seeded Solution Growth technique (TSSG) from nonstoichiometric liquid phases because BTO melts incongruently [8]. The raw materials were bismuth oxide (Bi203, Johnson Matthey, 99.995\%), titanium oxide (TiO2, Johnson Matthey, 99.995\%), terbium oxide (Sigma-Aldrich, 99.9\%), for terbium doped BT0 crystal (BTO:Tb), and niobium oxide (Nb205, Sigma-Aldrich, 99,99\%), for niobium doped BTO crystal (BTO:Nb). The liquid phases with compositions 10Bi203: xTi02:(1-x)/4Tb407 ( $\mathrm{x}=0.95$ and 0.90) for BTO:Tb and 10Bi203:xTiO2:(1-x)/2Nb205 ( $\mathrm{x}=0.95$ and 0.90) for BTO:Nb were used.

Crystals were grown using a resistive heating furnace equipped with a 2416 Eurotherm microprocessorbased digital temperature controller unit attached to a $\mathrm{Pt}-\mathrm{Pt} 10 \% \mathrm{Rh}$ thermocouple. Temperature fluctuations were lower than $0.2^{\circ} \mathrm{C}$ and the axial temperature gradient above the melt was of about $30^{\circ} \mathrm{C} / \mathrm{cm}$. BTO seeds oriented along the [001] direction were used attached to a pure platinum seed holder. All runs were carried out in air using pulling rates of $0.20 \mathrm{~mm} / \mathrm{h}$ and rotation rates of $30 \mathrm{rpm}$. Pulling and rotation rates were kept constant throughout each experiment and crystal diameter was visually controlled by changing the furnace temperature. After growth, the crystals were thermal annealed in temperatures between 700 and $750^{\circ} \mathrm{C}$ during about 72 hours to eliminate thermal stress. More details concerning crystals growing can be found in references $[3,9]$.

Crystals with dimensions of up to $80 \mathrm{~mm}$ in the axial direction and of about $13 \times 13 \mathrm{~mm}$ in the cross section were obtained. Intrinsic defects were observed in the initial part of grown crystals. A dark core was observed in the crystal shoulder, where the solid/liquid interface is convex. Some inclusions and voids appeared in the region of growth interface shape inversion from convex to planar, which occur after the crystal diameter reachs its stationary value. However, samples free of macroscopic defects, with volume up to $300 \mathrm{~mm}^{3}$, were cut from the middle part of crystals, and optically polished according to adequate crystallographic orientations. Figure 1 shows examples of as-grown crystals and polished samples of BTO:Tb and BTO:Nb crystals. 


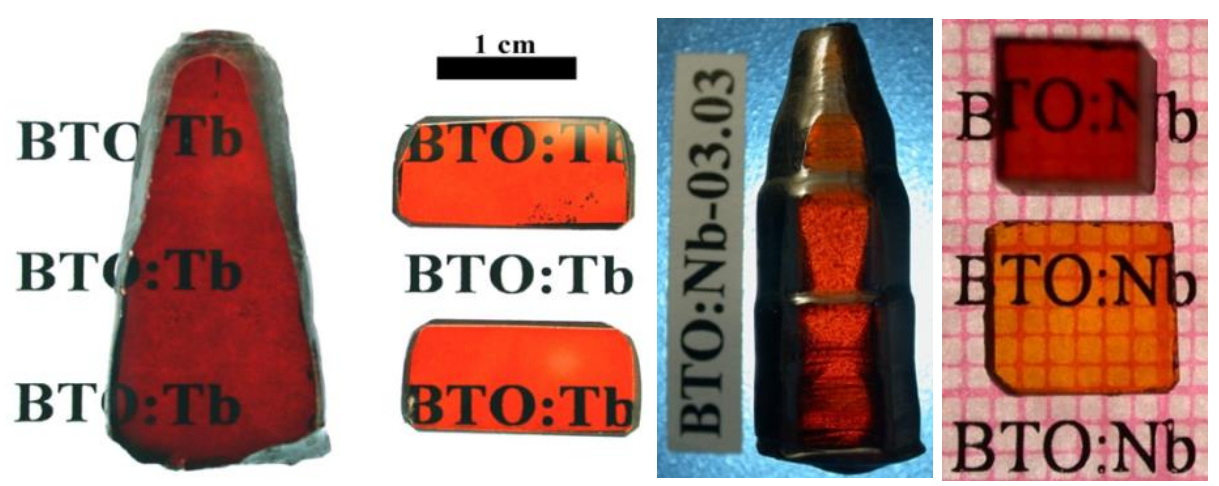

Fig. 1. BTO:Tb single crystals, on left, and BTO:Nb single crystals, on right.

\section{Optical Characterization}

Optical absorption spectra were taken at room temperature in the range of 700 to $400 \mathrm{~nm}$, using a Beckman DUß-70 Spectrophotometer. The absorption coefficient $\alpha$, taking in account multiple reflections, was calculated by the equation

$$
T=\frac{(1-R)^{2} e^{-\alpha d}}{1-R^{2} e^{-2 \alpha d}}
$$

where $T$ is the transmission coefficient, $d$ is the sample thickness, and $R$ is the reflection coefficient, calculated considering normal incidence and using the refractive index dispersion curve for pure BTO [8].

Figure 2 shows the absorption spectra of doped and undoped BTO crystals, for comparison. The optical absorption of doped crystals in the spectral range from about 2.6 to $3.1 \mathrm{eV}(477-400 \mathrm{~nm})$ is slightly smaller than that of undoped BTO. This behavior can be attributed to the lower concentration of the antisite $\left(B i_{M}^{3+}+h_{o}^{+}\right)$defect replacing partially $\mathrm{Ti}^{4+}$ in tetrahedral site. Such defect in undoped sillenite crystals is responsible for the absorption shoulder in this spectral region [10]. However, the crystal doping with 5+ or $3+$ ions modify this typical defects structure, probably by changing the anti-site $\operatorname{defect}\left(B i_{M}^{3+}+h_{o}^{+}\right)$to $B i_{M}^{3+}$, in the first case, or to $B i_{M}^{5+}$, in the second one [11]. One consequence is the reduction of the absorption shoulder in doped crystals. Also, the results suggest a decrease in the optical absorption of doped crystals from about 1.8 to $2.1 \mathrm{eV}(690-590 \mathrm{~nm})$ that can diminish the photosensitivity of crystals in this region.

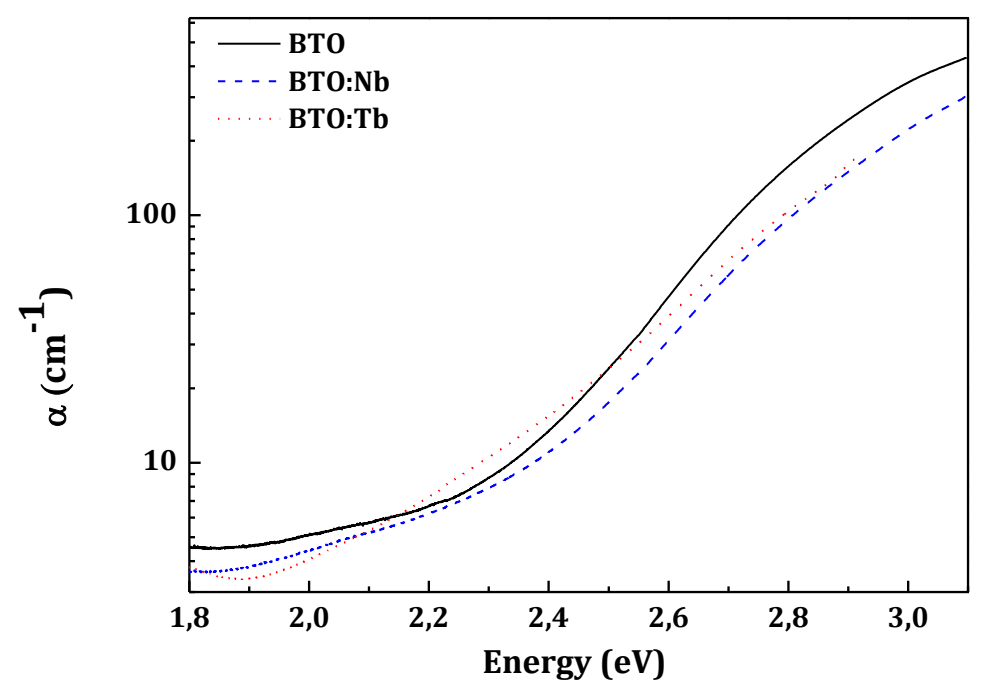

Fig. 2. Absorption coefficient $\alpha\left(\mathrm{cm}^{-1}\right)$ as a function energy (eV).

BTO is optically active, i.e. the polarization plane of linearly polarized light passing through the crystal is rotated by an angle $\Phi$. The specific rotation $\left(\rho_{o}\right)$ is used to specify this property being calculated by the 
ratio of the rotation angle and crystal thickness. The measurement procedure consists in placing the sample between crossed polarizers, illuminate it with expanded lasers beams, adjust the analyzer until extinction, and measure the rotation angle of the analyzer. Lasers at wavelength 632.8 and $543.5 \mathrm{~nm}$ were used in this work. Table 1 presents the measured $\rho_{o}$ values for doped and undoped crystals, showing that $\mathrm{Nb}$ doping increases the optical rotatory power by about $14 \%$, for $632.8 \mathrm{~nm}$, and $9 \%$, for $543.5 \mathrm{~nm}$, and $\mathrm{Tb}$ doping causes no change.

The electro-optical and photoconductivity measurements were carried out using a transverse configuration like shown in Fig. 3, where the general labels of sample dimensions are indicated.

TABLE 1. Properties of undoped BTO and doped with $\mathrm{Nb}$ and $\mathrm{Tb}$.

\begin{tabular}{lcccc}
\hline & $\begin{array}{c}\text { Energy }(\mathrm{eV}), \\
\text { Wavelength }(\mathrm{nm})\end{array}$ & BTO & BTO:Nb & BTO:Tb \\
\hline $\begin{array}{l}\text { Electro-optic coefficient, } \\
r_{41}(\mathrm{pm} / \mathrm{V})\end{array}$ & $1.96(632.8)$ & $(5.4 \pm 0.1)$ & $(5.9 \pm 0.1)$ & $(5.2 \pm 0.2)$ \\
\hline $\begin{array}{l}\text { Specific optical rotation, } \\
\rho_{0}(\% / \mathrm{mm})\end{array}$ & $1.96(632.8)$ & $(6.2 \pm 0.1)$ & $(7.1 \pm 0.1)$ & $(6.5 \pm 0.2)$ \\
\hline $\begin{array}{l}\text { Optical absorption } \\
\text { coefficient, } \alpha\left(\mathrm{cm}^{-1}\right)\end{array}$ & $1.96(632.8)$ & $4.13 .3 \pm 0.1)$ & $(11.2 \pm 0.1)$ & $(10.0 \pm 0.3)$ \\
\hline $\begin{array}{l}\text { Dark conductivity, } \sigma_{\text {dark }} \\
(\mathrm{A} / \mathrm{Vm})\end{array}$ & $2.28(543.5)$ & 7.49 & 4.80 & 3.68 \\
\hline
\end{tabular}

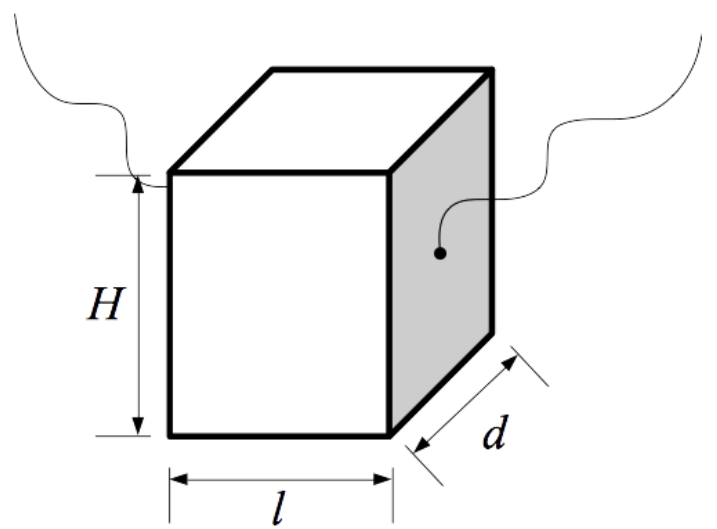

Fig. 3. Schematic representation of crystals samples used for electro-optical and photoconductivity measurements, where $H, d$ and $l$ are the height, thickness and distance between electrodes, respectively.

BTO is a cubic crystal with space group I23, so the electro-optic tensor has just three nonzero mutually equal components $r_{41}=r_{52}=r_{63}$. The values of $r_{41}$ for BTO:Tb and BTO:Nb were evaluated using the experimental procedure proposed by Henry et al. [12], which allows discriminate the effect of the optical activity from that of the field induced birefringence, necessary due to the high optical rotatory power of sillenite crystals. The experimental setup is schematically represented in Figure 4. The 632.8 and 543.5 $\mathrm{nm}$ lines of He-Ne lasers were used. Silver electrodes were deposited on $\{110\}$ faces, transverse to the light propagation. A dc voltage was applied across the crystal electrodes to generate the linear birefringence, changing the linearly polarized input light into an elliptically polarized one. The first linear polarizer is used to select the orientation of the input light polarization. For a fixed position of this polarizer, the analyzer is rotated to determine the maximum and minimum of the output intensities at the photodetector. These values are used to calculate the ellipticity as

$$
V_{\theta}=\frac{I_{\max }-I_{\min }}{I_{\max }+I_{\min }}
$$


The first polarizer is then rotate by $\pi / 4$ radians, the measurement procedure is repeated and the value of $V_{\theta+\pi / 4}$ is computed. This whole procedure is carried out for different values of applied dc voltage and the set of results are fitted to the equation

$$
V_{\theta}^{2}+V_{\theta+\pi / 4}^{2}=1+\left[1-\frac{\delta^{2}}{2}\left(\frac{\sin (\phi / 2)}{\phi / 2}\right)^{2}\right]^{2}
$$

where $\delta=\frac{2 \pi}{\lambda} n^{3} r_{41} E_{0} d, \phi^{2}=\rho^{2}+\delta^{2}, d$ is the crystal thickness, $n$ is the refractive index at the specific wavelength [8], $\rho$ is twice the rotation angle of the light polarization through the sample due to the optical activity, $E_{o}$ is calculated dividing the value of applied dc voltage by $l$, the distance between electrodes. The value of electro-optic coefficient $r_{41}$ is calculated from the fitting results. Samples with dimension $d=$ $3.8 \mathrm{~mm}, H=6.3 \mathrm{~mm}$ and $l=4.8 \mathrm{~mm}$ for BTO:Tb, and $d=3.4 \mathrm{~mm}, H=4.71 \mathrm{~mm}$ and $l=4.38 \mathrm{~mm}$ for BTO:Nb were used.
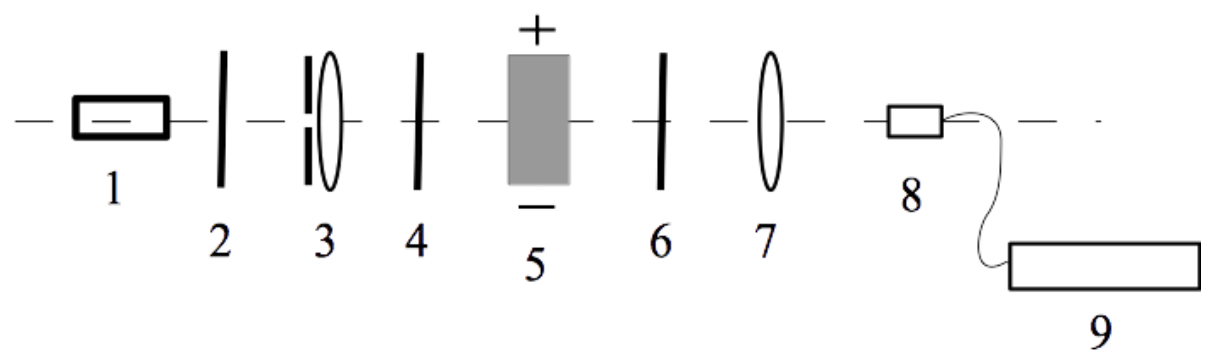

Fig. 4. Schematic representation of the experimental setup used for the electro-optic measurements. 1 . Laser light source; 2 . mechanical chopper; 3 . spatial filter and beam expander; 4. polarizer; 5 . crystal with silver electrodes on the (110) faces; 6 . analyzer; 7. lens to project the light in the front face of photodetector; 8. photodetector; 9 . lock-in amplifier.

The measured values of $r_{41}$ coefficients are shown in Table 1 . We can observe no dispersion of values for the wavelength tested, as expected according to ref. [13], but the doping with niobium produce an increase of about $9 \%$ in the value of $r_{41}$ in comparison with undoped crystal. Terbium doping do not affect these properties.

Wavelength-resolved photoconductivity experiments were carried out by illuminating the sample with white light-monochromator system and collecting the resulting photocurrent using electrodes on the transverse opposite lateral sides of the crystals, as shown in Figure 3. A dc voltage was applied between the electrodes and a Keithley System Electrometer Model 6514 was used to measure the current. The photoconductivity coefficient $\underline{\sigma}$ was calculated from [14]:

$$
\underline{\sigma}=\frac{l}{H} \frac{i_{p h}}{V} \frac{h v}{I(0)} \frac{\alpha}{1-e^{-\alpha d}}
$$

where $i_{p h}$ is the photocurrent, $V$ the transversely applied voltage, $h v$ is the photonic energy, $\alpha$ the absorption coefficient, $I(0)$ the irradiance at the input plane inside the sample, $l$ is the inter-electrode distance, $H$ and $d$ are height and thickness of crystal. In all wavelength-resolved photoconductivity experiments, it was used the absorption coefficient of BTO crystal. Dark conductivity was obtained by direct measurement of dc dark current $i_{\text {dark }}$ as a function of applied voltage $V$ using a Keithley System Electrometer Model 6514. All experiments were conducted at room temperature.

Figure 5 shows the results for the photoconductivity coefficient calculated by the Eq. 4, and dark conductivity values are shown in Table 1 . The effect of terbium doping on photoconductivity is negligible. However, the niobium doping increase both dark conductivity and photoconductivity by about 3 orders of magnitude. So, the $\mathrm{Nb}^{5+}$ ion changes in a significant way the structure of defects in the crystal, increasing the density of charge donors responsible for the dark conductivity and donors centers that can be photoexcited in a rate greater than in undoped crystal. This behavior combined with the suppression of absorption shoulder, discussed above, suggest that the $\mathrm{Nb}^{5+}$ ions compete with the generation of the antisite $\left(B i_{M}^{3+}+h_{o}^{+}\right)$, reducing its concentration and changing the mechanism of photogeneration of charge carriers in the doped crystals. The effect of this behavior on the photorefractive response of $\mathrm{Nb}$-doped crystal are being studied. 


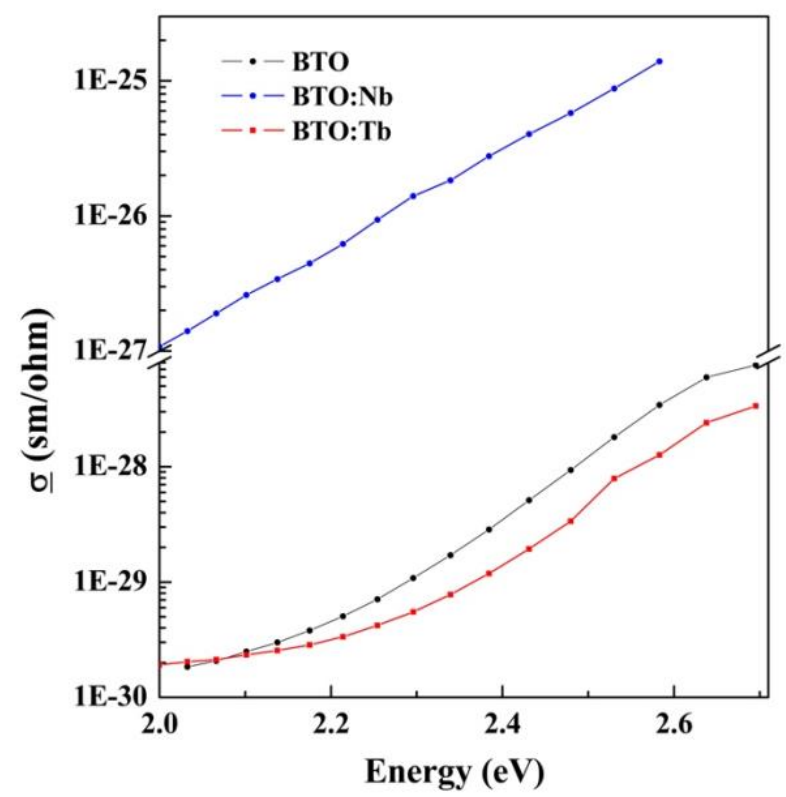

Fig. 5. Plot of normalized photoconductivity $\underline{\sigma}$ as a function of energy (eV) for applied fields parallel to BTO pure, BTO:Nb and BTO:Tb.

\section{Conclusions}

Terbium and niobium doped crystals with good crystalline quality were grown by TSSG technique. The results show that the doping with terbium ions do not cause significant changes in the investigated optical properties of crystals. However, the niobium doping causes important changes in the defects structure of crystals, modifying its photo-electrical response, affecting specially its photo and dark conductivity. The effect of such changes on the photorefractive behavior of Nb-doped BTO are in progress.

\section{Acknowledgements}

The financial support of the Brazilian research funding agencies FAPEG, CAPES, and CNPq is gratefully acknowledged. 